\title{
Genetic enhancement of macroautophagy in vertebrate models of neurodegenerative diseases
}

Patrick Ejlerskov ${ }^{*}, 1,2$, Avraham Ashkenazi ${ }^{*}, 1$ and David C. Rubinsztein ${ }^{1,3, \dagger}$

\author{
${ }^{1}$ University of Cambridge, Department of Medical Genetics, Cambridge Institute for Medical \\ Research, Wellcome/MRC Building, Addenbrooke's Hospital, Hills Road, Cambridge CB2 \\ OXY, UK \\ ${ }^{2}$ University of Copenhagen, Biotech Research and Innovation Centre, Ole Maaløes Vej 5, \\ 2200 Copenhagen N, Denmark \\ ${ }^{3}$ UK Dementia Research Institute, Cambridge Biomedical Campus, Cambridge Biomedical \\ Campus, Hills Road, Cambridge, UK. \\ ${ }^{*}$ Co-first authors \\ ${ }^{\dagger}$ For correspondence: dcr1000@cam.ac.uk
}

\begin{abstract}
Most of the neurodegenerative diseases that afflict humans manifest with the intraneuronal accumulation of toxic proteins which are aggregate-prone. Extensive data in cell and neuronal models support the concept that such proteins, like mutant huntingtin or alpha-synuclein, are substrates for macroautophagy (hereafter autophagy). Furthermore, autophagy-inducing compounds lower the levels of such proteins and ameliorate their toxicity in diverse animal models of neurodegenerative diseases. However, most of these compounds also have autophagy-independent effects and it is important to understand if similar benefits are seen with genetic strategies that upregulate autophagy, as this strengthens the validity of this strategy in such disease. Here we review studies in vertebrate models using genetic manipulations of core autophagy genes and describe how these improve pathology and neurodegeneration, this supporting the validity of autophagy upregulation as a target for certain neurodegenerative diseases.
\end{abstract}

Keywords: Autophagy, neurodegenerative disease, genetic therapy, beclin 1, ATG5, ATG7 


\section{Clearance of aggregate-prone proteins by autophagy}

Many neurodegenerative diseases such as Alzheimer's disease (AD), Huntington's diseases (HD), Parkinson's disease (PD) and Dementia with Lewy bodies (DLB) share the similar neuropathology of mutant protein misfolding and aggregation in the brain (Bancher et al., 1989; DiFiglia et al., 1997; Spillantini et al., 1998). In cells, mutant proteins associated with neurodegenerative diseases can form various oligomeric species and insoluble aggregates. We now know that many of the mutations confer new, toxic, gain-of-function properties on the proteins that eventually cause the death of specific neuronal populations in these diseases (Arrasate et al., 2004; Ashkenazi et al., 2017; DiFiglia et al., 1997). Thus, reduction in the levels of all species (soluble and insoluble) of the misfolded proteins can reduce the toxic burden in cells and prevent neuronal death (Berger et al., 2006; Ravikumar et al., 2002).

The ubiquitin-proteasome system and macroautophagy (hereafter autophagy) are the main degradative pathways within cells. Autophagy is an efficient process that clears cytoplasmic oligomeric species of the toxic proteins that are not accessible to the proteasome (Ciechanover and Kwon, 2015; Rubinsztein, 2006). Overall, autophagy induction shows beneficial effects by decreasing both the soluble and the insoluble forms of the misfolded proteins. Autophagy may also be able to reduce the levels of intraneuronal aggregates in some diseases, like spinocerebellar ataxia type 3, by enabling the removal of intracytoplasmic species which are in equilibrium with the mutant protein in the nucleus. Several molecules have been reported to induce autophagy and show beneficial effects in animal models of neurodegenerative diseases (Menzies et al., 2017; Rubinsztein et al., 2012). However, most of these compounds also have autophagy-independent effects and it is important to understand if similar benefits are seen with genetic strategies that upregulate autophagy, as this strengthen the validity of this strategy in such disease.

There is accumulating genetic support for the benefits of autophagy induction in vivo in models of neurodegeneration using mammalian autophagy proteins (ATG) that are involved in the synthesis of autophagosomes. In this review, we will briefly summarize small and nonsmall molecule inducers of autophagy and focus on autophagy genes that induce autophagy and reduce toxicity in animal models of $\mathrm{AD}, \mathrm{HD}$, and $\mathrm{PD}$.

\section{Key autophagy genes for autophagosome synthesis}

The first morphologically recognisable structure in autophagy is a double-layered, cupshaped membrane structure called a phagophore that elongates and engulfs cytoplasmic cargo 
such as aggregate-prone proteins. After the edges of the phagophores are closed to form vesicular autophagosomes, these traffic to and fuse with lysosomes and thereby form autolysosomes, in which the luminal content is degraded by lysosomal enzymes (Mizushima et al., 2008). There are several key ATG that regulate this process (Figure 1).

Upon autophagy induction by nutrient deprivation (an evolutionary conserved autophagy stimulus), the class III VPS34 is recruited to nascent phagophore membranes. In a complex with ATG6 (beclin 1), VPS15, and ATG14, class III VPS34 generates phosphatidylinositol 3phosphate (PI3P) (Tassa et al., 2003). PI3P on autophagosomal precursor membranes recruits PI3P effectors such as WD repeat domain phosphoinositide-interacting protein 2 (WIPI2). This enables WIPI2 to interact with other downstream autophagy effectors, such as ATG16L1, which regulate the formation of autophagosomes (Ashkenazi et al., 2017; Dooley et al., 2014; Vicinanza et al., 2015).

ATG16L1 acts in a complex with ATG12 and ATG5. ATG12, an ubiquitin like molecule, is conjugated to ATG5 by the E1- and E2-like enzymes ATG7 and ATG10, respectively. Subsequently, ATG12-ATG5 binds non-covalently to ATG16L1. The interaction of the ATG12-ATG5-ATG16L1 complex with pre-autophagosomal membranes assist in the recruitment of members of the Atg8 family, like LC3, another ubiquitin-like protein. This is achieved via the conjugation of LC3-I to phosphatidylethanolamine which thereby generates lipidated LC3B-II on autophagosomal membranes. This reaction is governed by ATG7, ATG3, and ATG12-ATG5-ATG16L1, acting as E1-, E2-, and E3-like enzymes, respectively. The Atg8/LC3 family are thought to promote phagopore elongation and closure to generate a complete autophagosome vesicle (Bento et al., 2016). Specific knock-out of Atg5 and Atg7 in neurons causes accumulation of neuronal aggregates (Hara et al., 2006; Komatsu et al., 2006), emphasising the crucial importance of autophagy in maintaining normal neuronal homeostasis.

\section{Main regulatory pathways of autophagosome synthesis}

In normal physiological conditions, autophagosome synthesis occurs at a basal level, but the autophagy flux can further increased upon nutrient deprivation. This is partially governed by inhibition of mammalian target of rapamycin (mTOR), a serine/threonine kinase, which interacts with partner proteins in a larger complex termed the mTOR complex 1 (mTORC1). mTORC1 and AMP-dependent protein kinase (AMPK) sense intracellular levels of energy (e.g. AMP/ATP ratio), nutrients, and growth factors, and control the activity of the 
mammalian Atg1 homologues UNC-51-like kinase (ULK1/2), a serine/threonine-protein kinase (Kim et al., 2011). ULK1/2 resides in a complex with ATG101, ATG13, and focal adhesion kinase family interacting protein of $200 \mathrm{kD}$ (FIP200). Activation of the ULK complex regulates the efficient recruitment of the class III VPS34 complex to preautophagosomal membranes to initiate autophagosome synthesis, as described above. A chain of phosphorylation events controls the activation of the ULK complex. In this regard, AMPK is a positive regulator of the ULK complex, while mTORC1 is a negative regulator (Ganley et al., 2009; Laplante and Sabatini, 2012). Neuronal knockouts of Fip200 and Ulk1/2 in mice cause progressive degeneration in different parts of the brain (Joo et al., 2016; Liang et al., 2010). Another interaction that regulates autophagy at the level of the VPS34 complex is via B-cell lymphoma 2 (BCL2). BCL2 inhibits autophagy through an interaction with the BH3 domain of the beclin 1 , and this association is disrupted by nutrient deprivation-induced autophagy (He et al., 2012).

Autophagosome synthesis is also transcriptionally regulated. Under normal physiological conditions, mTORC1 phosphorylates transcription factor EB (TFEB), which keeps TFEB in the cytosol (Settembre et al., 2012). Under nutrient-deprived conditions where mTORC1 is inactive, TFEB is no longer phosphorylated and translocates to the nucleus, where it initiates transcription of many genes directly involved in the biogenesis of lysosomes and the synthesis of autophagosomes (Roczniak-Ferguson et al., 2012; Settembre et al., 2011).

\section{Small molecule inducers of autophagy in animal models of neurodegeneration}

Several small molecule inducers of autophagy affect upstream regulatory pathways of autophagosome synthesis, such as mTOR. Rapamycin and other rapalogs are non-ATP competitive inhibitors of mTOR, with selectivity towards mTORC1 (Kim and Guan, 2015). These compounds are autophagy inducers and have beneficial effects in several animal models of AD, PD, frontotemporal dementia (FTD), prion diseases (PrP), and HD (Cortes et al., 2012; Jiang et al., 2014; Menzies et al., 2010; Ozcelik et al., 2013; Ravikumar et al., 2004; Sarkar et al., 2009; Spilman et al., 2010; Wang et al., 2012). However, there is a growing list of molecules that act on targets independent of mTOR. One such target is AMPK, which recently was discovered to be the target of another potent autophagy inducer, trehalose, a molecule that has been well-studied in vivo (DeBosch et al., 2016). This disaccharide induces autophagy and ameliorates toxicity in animal models of AD, FTD, PD, HD, spinocerebellar ataxia type 17 (SCA17), amyotrophic lateral sclerosis (ALS), and oculopharyngeal muscular dystrophy (OPMD) (Aguib et al., 2009; Castillo et al., 2013; Chen 
et al., 2015; Davies et al., 2006; Li et al., 2015; Rodriguez-Navarro et al., 2010; Schaeffer and Goedert, 2012; Tanaka et al., 2004; Tanji et al., 2015). Other AMPK activators, such as metformin (Berthier et al., 2016; Ma et al., 2007; Son et al., 2016) and nilotinib (Hebron et al., 2014; Lonskaya et al., 2015; Yu et al., 2013), also show beneficial effects in models of neurodegeneration.

Another group of compounds act on cyclic AMP (cAMP) and inositol triphosphate $\left(\mathrm{IP}_{3}\right)$ pathways, independently of mTOR (Williams et al., 2008). While $\mathrm{IP}_{3}$ impacts many processes, it also negatively regulates autophagy. Lithium administration prevents inositol recycling and reduces $\mathrm{IP}_{3}$ levels, which induces autophagy, and has been shown to be beneficial in an animal model of AD (Zhang et al., 2011). Additional compounds that seem to act on this cAMP-IP 3 autophagy pathway are rilmenidine, minoxidil, clonidine, and verapamil (Rose et al., 2010; Williams et al., 2008). It should be noted that these compounds may have other targets that are not autophagy related. Rilmenidine was recently tested in an open-label study to assess the feasibility and tolerability of the drug for HD patients (Underwood et al., 2017).

\section{Gene therapy approaches using core autophagy genes in models of neurodegenerative} disease

While studies of the aforementioned compounds suggest that autophagy upregulation is beneficial in many animal models of neurodegenerative disease, these molecules and their targets may also impact pathways distinct from autophagy. Thus, genetic studies manipulating autophagy proteins allow an alternative and potentially more specific way to validate autophagy as a target for some of these conditions. Virally-mediated gene therapy using beclin 1 (Becn1) has been the most frequently used strategy for such objectives and has benefits in animal models of neurodegenerative disease such as AD (Pickford et al., 2008; Rocchi et al., 2017), PD (Spencer et al., 2009), and polyglutamine diseases (NascimentoFerreira et al., 2011). Additionally, both ATG5 and ATG7 have been reported to have neuroprotective properties in in vivo models of neurodegeneration (Crews et al., 2010; Lopez et al., 2017). In the following section we will elaborate on some recent studies which have used core autophagy genes to rescue animal models of neurodegenerative disease. 


\section{Beclin 1}

\section{Alzheimer's disease}

Pickford and co-workers showed neurobeneficial effects of beclin 1 expression in a mouse model of AD (Pickford et al., 2008). The authors used the transgenic T41 mice expressing amyloid precursor protein (APP) with both the Swedish (K670N/M671L) and the London (V717I) mutations, which develop amyloid plaques in the frontal cortex, hippocampus, thalamus, and the olfactory region associated with an age-dependent decline in learning and memory skills (Rockenstein et al., 2001). Crossing T41 APP heterozygous mice with $B e c n 1^{+/-}$mice (Becn1 $1^{-/-}$is prenatal lethal) caused increased accumulation of amyloid- $\beta$ (A $\beta$ ) immunoreactivity in the hippocampus and frontoparietal cortex of 9-month-old mice compared to age-matched T41 $\mathrm{APP}^{+}$mice. The accumulation of $\mathrm{A} \beta$ was further confirmed by ELISA of formic acid-extracted A $\beta$ species, which was inversely correlated with the levels of beclin 1 in the mice. Electron microscopy analysis of neurons from 5-months-old mice revealed abnormally enlarged lysosomes containing electron dense material, and western blotting of cortical tissue from 16-months-old $\mathrm{APP}^{+} \mathrm{x} \mathrm{Becn}^{+/-}$-crossed mice showed reduced LC3II/I conversion ratio by western blotting. Additionally, in the prefrontal cortex of 9months-old $\mathrm{APP}^{+} \mathrm{x} \mathrm{Becn}^{+-}$mice, the authors observed increased numbers of activated CD68 ${ }^{+}$ microglia, the innate immune cells of the brain, which was associated with loss of synaptodendritic integrity, an early indicator of ongoing neurodegeneration. Conversely, expressing beclin 1 via lentiviral transduction in hippocampus and cortex of $\mathrm{APP}^{+}$mice significantly reduced accumulation of $A \beta$ and thioflavin-positive aggregates, when compared to the contralateral side of the brain only receiving GFP control lentivirus.

These findings have been expanded by a recent study using an elegant new mouse model expressing a knock-in mutant F121A in Becn1, which prevents BCL2 binding to the BH3 domain of beclin 1 and consequently decreases its inhibitory effect on autophagy (Rocchi et al., 2017). These mice have hyperactive autophagy flux indicated by increased LC3-II levels and reduced p62 levels. Injecting the lysosomal inhibitor chloroquine to the mice even further increased the LC3-II levels, underscoring an increased autophagy flux in the Becn $1^{F 121 A}$ mice. These mice were crossed with $5 x F A D$ transgenic $\mathrm{AD}$ mice, which express 5 familial mutations in human APP and presenilin (PS1) genes, which causes amyloid plaques at an age of 2 months and cognitive decline after 6 months (Oakley et al., 2006). The Beclin ${ }^{F 121 A}$ mice crossed with 5xFAD mice have reduced accumulation of soluble as well as insoluble $A \beta_{42}$, reduced thioflavin-positive aggregates in the cortex, had longer survival rates, and performed 
significantly better in the Morris water maze than the 5xFAD age-matched mice. Thus, promoting autophagy via beclin 1 is neuroprotective in various models of $\mathrm{AD}$.

\section{Parkinson's disease}

Beclin 1 also is protective in models of PD (Spencer et al., 2009), for example in transgenic mice expressing $\alpha$-synuclein under the neuronal specific PDGF- $\beta$ promoter. These mice experience neuronal accumulation of aggregated $\alpha$-synuclein, loss of dopaminergic neurons, and lysosomal abnormalities, which is associated with behavioural defects (Masliah et al., 2000). In vivo lentiviral expression of beclin 1 in temporal cortex and hippocampus of the $\alpha$ synuclein transgenic mice increased LC3 immunoreactivity in these areas, which was associated with lowering of the neuronal $\alpha$-synuclein burden. In beclin 1 expressing mice $\alpha$ synuclein were more frequently found in vesicular structures in neurons indicative of autophagosomal processing of the pathogenic protein. This was accompanied with preservation of the neuronal network, indicated by sustained immunoreactivity for synaptophysin and MAP2 in the brain area near the injection sites, when compared to control injected mice.

\section{Polyglutamine diseases}

Beclin 1 overexpression also has beneficial consequences in a model of Machado-Joseph disease (MJD)/SCA3, an autosomal dominant disease caused by a polyglutamine (polyQ) expansion encoded by an elongated CAG trinucleotide repeat mutation in the coding region of the ataxin-3 gene (Kawaguchi et al., 1994). Post-mortem brain tissues and fibroblasts from MJD patients have lower levels of beclin 1 (Ashkenazi et al., 2017; Nascimento-Ferreira et al., 2013; Sittler et al., 2017). The polyQ stretch in wild-type ataxin-3 allows it to bind and deubiquitinate beclin 1 (Ashkenazi et al., 2017). Thus, ataxin-3 can protect beclin 1 from proteasome-mediated degradation and thereby promote autophagy. Expansion of the polyQ tract in ataxin-3 decreases its deubiquitinase activity toward beclin-1 in vitro, which destabilizes beclin 1 levels (Ashkenazi et al., 2017). Accordingly, lentiviral beclin 1 overexpression attenuated mutant ataxin-3-induced pathology in the striatum of a rat model of SCA3 (Nascimento-Ferreira et al., 2011). The beclin 1 expression significantly reduced the number of mutant ataxin-3-positive aggregates. Similar benefits were observed with lentiviral overexpression of beclin 1 in a transgenic mouse model of this disease (Nascimento-Ferreira et al., 2013). 


\section{ATG5}

\section{Alzheimer's disease}

The rare p.A153T variant of the microtubule associated protein tau (MAPT) is associated with increased risk of FTD and AD (Coppola et al., 2012). In order to study this tau variant in vivo, an experimental zebrafish model was generated expressing wild-type tau or mutant $\operatorname{tau}_{\mathrm{A} 153 \mathrm{~T}}$ both coupled to the photoactivatable fluorophore Dendra (Lopez et al., 2017). The $\operatorname{tau}_{\mathrm{A} 152 \mathrm{~T}}$ zebrafish showed morphological abnormalities in the spinal cord, reduced motor neuron numbers, and accordingly had impaired escape responses. Additionally, the mutant fish had increased levels of pathogenic hyperphosphorylated tau, which was associated with reduced degradation kinetics of the tau protein. While the autophagy flux was unaffected in the zebrafish with the $\operatorname{tau}_{\mathrm{A} 153 \mathrm{~T}}$ variant, proteasomal clearance was impaired, indicated by reduced degradation of the proteasome-specific substrate UbG76V-GFP (Dantuma et al., 2000). As tau is known to be a substrate for both proteasome and autophagy degradation (Lee et al., 2013), we tested whether promoting the autophagy pathway could reverse the accumulation of tau and morphological defects observed in the tau $\mathrm{A}_{\mathrm{A} 53 \mathrm{~T}}$ zebrafish. Both chemically and genetic promotion of autophagy rescued the pathology observed in the zebrafish. The latter was accomplished by transient expression of the autophagy gene Atg5, which upregulated autophagy and ameliorated the abnormal morphology of the zebrafish carrying the $\operatorname{tau}_{\mathrm{A} 153 \mathrm{~T}}$ variant.

\section{ATG7}

Brain homogenates from post-mortem patients with dementia with Lewy bodies (DLB) or from mice overexpressing human wild-type $\alpha$-synuclein revealed downregulation of ATG7 and upregulation of the negative autophagy regulator mTOR (Crews et al., 2010). Interestingly, lentiviral-mediated overexpression of ATG7 in the cortex and the hippocampus of $\alpha$-synuclein transgenic mice significantly reduced the $\alpha$-synuclein burden while preserving the neuronal network indicated by MAP2 staining.

\section{Other autophagy related genes}

Expression of upstream activators of autophagy has also shown to be neuroprotective in several models of neurodegenerative disorders. Viral overexpression of TFEB in the striatum of HD mice (HDQ175/Q7) promoted autophagy and reduced levels of the disease causing protein, mutant huntingtin (Vodicka et al., 2016). Similarly, viral-mediated expression of TFEB was beneficial in a rat model of PD expressing human $\alpha$-synuclein, causing a 
significant reduction in the $\alpha$-synuclein load, accompanied by preservation of dopaminergic neurons (Decressac et al., 2013).

In conclusion, genetic manipulation of both core autophagy genes but also close regulators of the autophagy pathway is neuroprotective in various in vivo models of neurodegeneration. These studies provide strong support along with the existing literature on chemical stimulators of autophagy, that specific upregulation of this pathway is beneficial in various models of neurodegenerative diseases. These genetic studies also allow assessment of the safety of this strategy - e.g. no side effects were reported in the Becn1 ${ }^{F 121 A}$ mice and ATG5 transgenic mice have increased lifespans and antiageing phenotypes (Pyo et al., 2013). In addition, genetic strategies may be promising avenues to pursue for human therapeutics. For example, the autophagy-inducing beclin 1 peptide strategy described by Levine and colleagues (Shoji-Kawata et al., 2013) suggests approaches distinct from conventional small molecules that may have utility in diseases where autophagy upregulation may be advantageous. Finally, it is important to bear in mind that while induction of autophagosome biogenesis may be beneficial in many neurodegenderative diseases, it may not be effective and could possibly be deleterious when autophagy flux is impaired after autophagosomes are formed, for example by mutations affecting lysosomal activity. Thus, understanding the molecular basis of disease will be an important adjunct to this and many other therapeutic approaches. 


\section{Funding Statement}

This work was supported Strategic Grant to Cambridge Institute for Medical Research [grant number 100140/Z/12/Z], UK Dementia Research Institute (funded by the MRC, Alzheimers Research UK and the Alzheimer's Society) (DCR), The Tau Consortium, Alzheimer's Research UK, Astra Zeneca, Danish Council for Independent Research [grant number 611000461B], Lundbeck Foundation [grant number R210-2015-3372], and Parkinsonforeningen.

\section{Competing financial interests}

D.C.R consults for E3Bio and Astra Zeneca. 


\section{Reference list}

Aguib, Y., et al., 2009. Autophagy induction by trehalose counteracts cellular prion infection. Autophagy. 5, 361-9.

Arrasate, M., et al., 2004. Inclusion body formation reduces levels of mutant huntingtin and the risk of neuronal death. Nature. 431, 805-10.

Ashkenazi, A., et al., 2017. Polyglutamine tracts regulate beclin 1-dependent autophagy. Nature. 545, 108-111.

Bancher, C., et al., 1989. Accumulation of abnormally phosphorylated tau precedes the formation of neurofibrillary tangles in Alzheimer's disease. Brain Res. 477, 90-9.

Bento, C. F., et al., 2016. Mammalian Autophagy: How Does It Work? Annu Rev Biochem. 85, 685713.

Berger, Z., et al., 2006. Rapamycin alleviates toxicity of different aggregate-prone proteins. Hum Mol Genet. 15, 433-42.

Berthier, A., et al., 2016. Pharmacological Interventions to Ameliorate Neuropathological Symptoms in a Mouse Model of Lafora Disease. Mol Neurobiol. 53, 1296-309.

Castillo, K., et al., 2013. Trehalose delays the progression of amyotrophic lateral sclerosis by enhancing autophagy in motoneurons. Autophagy. 9, 1308-20.

Chen, Z. Z., et al., 2015. Trehalose attenuates the gait ataxia and gliosis of spinocerebellar ataxia type 17 mice. Neurochem Res. 40, 800-10.

Ciechanover, A., Kwon, Y. T., 2015. Degradation of misfolded proteins in neurodegenerative diseases: therapeutic targets and strategies. Exp Mol Med. 47, e147.

Coppola, G., et al., 2012. Evidence for a role of the rare p.A152T variant in MAPT in increasing the risk for FTD-spectrum and Alzheimer's diseases. Hum Mol Genet. 21, 3500-12.

Cortes, C. J., et al., 2012. Rapamycin delays disease onset and prevents PrP plaque deposition in a mouse model of Gerstmann-Straussler-Scheinker disease. J Neurosci. 32, 12396-405.

Crews, L., et al., 2010. Selective molecular alterations in the autophagy pathway in patients with Lewy body disease and in models of alpha-synucleinopathy. PLoS One. 5, e9313.

Dantuma, N. P., et al., 2000. Short-lived green fluorescent proteins for quantifying ubiquitin/proteasome-dependent proteolysis in living cells. Nat Biotechnol. 18, 538-43.

Davies, J. E., et al., 2006. Trehalose reduces aggregate formation and delays pathology in a transgenic mouse model of oculopharyngeal muscular dystrophy. Hum Mol Genet. 15, 2331.

DeBosch, B. J., et al., 2016. Trehalose inhibits solute carrier 2A (SLC2A) proteins to induce autophagy and prevent hepatic steatosis. Sci Signal. 9, ra21.

Decressac, M., et al., 2013. TFEB-mediated autophagy rescues midbrain dopamine neurons from alpha-synuclein toxicity. Proc Natl Acad Sci U S A. 110, E1817-26.

DiFiglia, M., et al., 1997. Aggregation of huntingtin in neuronal intranuclear inclusions and dystrophic neurites in brain. Science. 277, 1990-3.

Dooley, H. C., et al., 2014. WIPI2 links LC3 conjugation with PI3P, autophagosome formation, and pathogen clearance by recruiting Atg12-5-16L1. Mol Cell. 55, 238-52.

Ganley, I. G., et al., 2009. ULK1.ATG13.FIP200 complex mediates mTOR signaling and is essential for autophagy. J Biol Chem. 284, 12297-305.

Hara, T., et al., 2006. Suppression of basal autophagy in neural cells causes neurodegenerative disease in mice. Nature. 441, 885-9.

He, C., et al., 2012. Exercise-induced BCL2-regulated autophagy is required for muscle glucose homeostasis. Nature. 481, 511-5.

Hebron, M. L., et al., 2014. Tyrosine Kinase Inhibition Regulates Early Systemic Immune Changes and Modulates the Neuroimmune Response in alpha-Synucleinopathy. J Clin Cell Immunol. 5, 259. 
Jiang, T., et al., 2014. Temsirolimus attenuates tauopathy in vitro and in vivo by targeting tau hyperphosphorylation and autophagic clearance. Neuropharmacology. 85, 121-30.

Joo, J. H., et al., 2016. The Noncanonical Role of ULK/ATG1 in ER-to-Golgi Trafficking Is Essential for Cellular Homeostasis. Mol Cell. 62, 491-506.

Kawaguchi, Y., et al., 1994. CAG expansions in a novel gene for Machado-Joseph disease at chromosome 14q32.1. Nat Genet. 8, 221-8.

Kim, J., et al., 2011. AMPK and mTOR regulate autophagy through direct phosphorylation of Ulk1. Nat Cell Biol. 13, 132-41.

Kim, Y. C., Guan, K. L., 2015. mTOR: a pharmacologic target for autophagy regulation. J Clin Invest. 125, 25-32.

Komatsu, M., et al., 2006. Loss of autophagy in the central nervous system causes neurodegeneration in mice. Nature. 441, 880-4.

Laplante, M., Sabatini, D. M., 2012. mTOR signaling in growth control and disease. Cell. 149, 27493.

Lee, M. J., et al., 2013. Tau degradation: the ubiquitin-proteasome system versus the autophagylysosome system. Prog Neurobiol. 105, 49-59.

$\mathrm{Li}, \mathrm{Y}$., et al., 2015. Trehalose decreases mutant SOD1 expression and alleviates motor deficiency in early but not end-stage amyotrophic lateral sclerosis in a SOD1-G93A mouse model. Neuroscience. 298, 12-25.

Liang, C. C., et al., 2010. Neural-specific deletion of FIP200 leads to cerebellar degeneration caused by increased neuronal death and axon degeneration. J Biol Chem. 285, 3499-509.

Lonskaya, l., et al., 2015. Nilotinib and bosutinib modulate pre-plaque alterations of blood immune markers and neuro-inflammation in Alzheimer's disease models. Neuroscience. 304, 316-27.

Lopez, A., et al., 2017. A152T tau allele causes neurodegeneration that can be ameliorated in a zebrafish model by autophagy induction. Brain. 140, 1128-1146.

Ma, T. C., et al., 2007. Metformin therapy in a transgenic mouse model of Huntington's disease. Neurosci Lett. 411, 98-103.

Masliah, E., et al., 2000. Dopaminergic loss and inclusion body formation in alpha-synuclein mice: implications for neurodegenerative disorders. Science. 287, 1265-9.

Menzies, F. M., et al., 2017. Autophagy and Neurodegeneration: Pathogenic Mechanisms and Therapeutic Opportunities. Neuron. 93, 1015-1034.

Menzies, F. M., et al., 2010. Autophagy induction reduces mutant ataxin-3 levels and toxicity in a mouse model of spinocerebellar ataxia type 3. Brain. 133, 93-104.

Mizushima, N., et al., 2008. Autophagy fights disease through cellular self-digestion. Nature. 451, 1069-75.

Nascimento-Ferreira, l., et al., 2013. Beclin 1 mitigates motor and neuropathological deficits in genetic mouse models of Machado-Joseph disease. Brain. 136, 2173-88.

Nascimento-Ferreira, l., et al., 2011. Overexpression of the autophagic beclin-1 protein clears mutant ataxin-3 and alleviates Machado-Joseph disease. Brain. 134, 1400-15.

Oakley, H., et al., 2006. Intraneuronal beta-amyloid aggregates, neurodegeneration, and neuron loss in transgenic mice with five familial Alzheimer's disease mutations: potential factors in amyloid plaque formation. J Neurosci. 26, 10129-40.

Ozcelik, S., et al., 2013. Rapamycin attenuates the progression of tau pathology in P301S tau transgenic mice. PLoS One. 8, e62459.

Pickford, F., et al., 2008. The autophagy-related protein beclin 1 shows reduced expression in early Alzheimer disease and regulates amyloid beta accumulation in mice. J Clin Invest. 118, 2190-9.

Pyo, J. O., et al., 2013. Overexpression of Atg5 in mice activates autophagy and extends lifespan. Nat Commun. 4, 2300. 
Ravikumar, B., et al., 2002. Aggregate-prone proteins with polyglutamine and polyalanine expansions are degraded by autophagy. Hum Mol Genet. 11, 1107-17.

Ravikumar, B., et al., 2004. Inhibition of mTOR induces autophagy and reduces toxicity of polyglutamine expansions in fly and mouse models of Huntington disease. Nat Genet. 36, 585-95.

Rocchi, A., et al., 2017. A Becn1 mutation mediates hyperactive autophagic sequestration of amyloid oligomers and improved cognition in Alzheimer's disease. PLoS Genet. 13, e1006962.

Rockenstein, E., et al., 2001. Early formation of mature amyloid-beta protein deposits in a mutant APP transgenic model depends on levels of Abeta(1-42). J Neurosci Res. 66, 573-82.

Roczniak-Ferguson, A., et al., 2012. The transcription factor TFEB links mTORC1 signaling to transcriptional control of lysosome homeostasis. Sci Signal. 5, ra42.

Rodriguez-Navarro, J. A., et al., 2010. Trehalose ameliorates dopaminergic and tau pathology in parkin deleted/tau overexpressing mice through autophagy activation. Neurobiol Dis. 39, 423-38.

Rose, C., et al., 2010. Rilmenidine attenuates toxicity of polyglutamine expansions in a mouse model of Huntington's disease. Hum Mol Genet. 19, 2144-53.

Rubinsztein, D. C., 2006. The roles of intracellular protein-degradation pathways in neurodegeneration. Nature. 443, 780-6.

Rubinsztein, D. C., et al., 2012. Autophagy modulation as a potential therapeutic target for diverse diseases. Nat Rev Drug Discov. 11, 709-30.

Sarkar, S., et al., 2009. Rapamycin and mTOR-independent autophagy inducers ameliorate toxicity of polyglutamine-expanded huntingtin and related proteinopathies. Cell Death Differ. 16, 46-56.

Schaeffer, V., Goedert, M., 2012. Stimulation of autophagy is neuroprotective in a mouse model of human tauopathy. Autophagy. 8, 1686-7.

Settembre, C., et al., 2011. TFEB links autophagy to lysosomal biogenesis. Science. 332, 1429-33.

Settembre, C., et al., 2012. A lysosome-to-nucleus signalling mechanism senses and regulates the Iysosome via mTOR and TFEB. EMBO J. 31, 1095-108.

Shoji-Kawata, S., et al., 2013. Identification of a candidate therapeutic autophagy-inducing peptide. Nature. 494, 201-6.

Sittler, A., et al., 2017. Deregulation of autophagy in postmortem brains of Machado-Joseph disease patients. Neuropathology.

Son, S. M., et al., 2016. Metformin Facilitates Amyloid-beta Generation by beta- and gammaSecretases via Autophagy Activation. J Alzheimers Dis. 51, 1197-208.

Spencer, B., et al., 2009. Beclin 1 gene transfer activates autophagy and ameliorates the neurodegenerative pathology in alpha-synuclein models of Parkinson's and Lewy body diseases. J Neurosci. 29, 13578-88.

Spillantini, M. G., et al., 1998. alpha-Synuclein in filamentous inclusions of Lewy bodies from Parkinson's disease and dementia with lewy bodies. Proc Natl Acad Sci U S A. 95, 6469-73.

Spilman, P., et al., 2010. Inhibition of mTOR by rapamycin abolishes cognitive deficits and reduces amyloid-beta levels in a mouse model of Alzheimer's disease. PLoS One. 5, e9979.

Tanaka, M., et al., 2004. Trehalose alleviates polyglutamine-mediated pathology in a mouse model of Huntington disease. Nat Med. 10, 148-54.

Tanji, K., et al., 2015. Trehalose intake induces chaperone molecules along with autophagy in a mouse model of Lewy body disease. Biochem Biophys Res Commun. 465, 746-52.

Tassa, A., et al., 2003. Class III phosphoinositide 3-kinase--Beclin1 complex mediates the amino acid-dependent regulation of autophagy in C2C12 myotubes. Biochem J. 376, 577-86.

Underwood, B. R., et al., 2017. An open-label study to assess the feasibility and tolerability of rilmenidine for the treatment of Huntington's disease. J Neurol.

Vicinanza, M., et al., 2015. PI(5)P regulates autophagosome biogenesis. Mol Cell. 57, 219-34. 
Vodicka, P., et al., 2016. Autophagy Activation by Transcription Factor EB (TFEB) in Striatum of HDQ175/Q7 Mice. J Huntingtons Dis. 5, 249-260.

Wang, I. F., et al., 2012. Autophagy activators rescue and alleviate pathogenesis of a mouse model with proteinopathies of the TAR DNA-binding protein 43. Proc Natl Acad Sci U S A. 109, 15024-9.

Williams, A., et al., 2008. Novel targets for Huntington's disease in an mTOR-independent autophagy pathway. Nat Chem Biol. 4, 295-305.

$\mathrm{Yu}, \mathrm{H}$. C., et al., 2013. Nilotinib induces autophagy in hepatocellular carcinoma through AMPK activation. J Biol Chem. 288, 18249-59.

Zhang, X., et al., 2011. Long-term treatment with lithium alleviates memory deficits and reduces amyloid-beta production in an aged Alzheimer's disease transgenic mouse model. J Alzheimers Dis. 24, 739-49. 


\section{Figure legend}

Figure 1. Schematic overview of selected core autophagy proteins and their involvement in autophagy. The formation of an autophagosome is initiated by activation of the ULK complex (ULK1/2, ATG13, and FIP200) which subsequently 1) recruits the beclin 1, VPS34, VPS15 and ATG14L complex. VPS34 generates phosphatidylinositol 3-phosphate (PI3P) at the phagophore membrane. 2) The levels of beclin 1 are regulated by ubiquitination via E3 ligases which target it for 3) proteasomal degradation. 2) Conversely, deubiquitinases (DUB), e.g. Ataxin-3, deubiquitinates beclin 1 and thereby enables it to associate with VPS34 complexes and to induce autophagy. 4) PI3P facilitates recruitment of WIPI2 to the phagophore, which subsequently interacts with the ATG16L1/ATG5-ATG12 complex. 5) Pro-LC3 is cleaved by ATG4 to form cytosolic LC3-I, which interacts with ATG7 and subsequently ATG3. 6) ATG7 acts as an E1-like enzyme and couples LC3-I to the E2-like enzyme ATG3. This enables the E3-like complex of ATG16L1/ATG5-ATG12 to conjugate the ubiquitin-like LC3-I to phosphatidylethanolamine in phagophore membranes, thereby forming lipidated LC3-II. 7) The recruitment to autophagosomes of some cytosolic cargoes, like aggregate-prone proteins, is enhanced by autophagy receptors e.g. SQSTM1/p62, which link these substrates to LC3-II. 8) Eventually, the phagophore membrane closes to form a vesicular autophagosome which 9) fuses with low-pH lysosomes. Degradative enzymes from the lysosome will degrade the luminal content of autophagsomes. 


\section{Nucleus}

\section{Proteasomal}

degradation

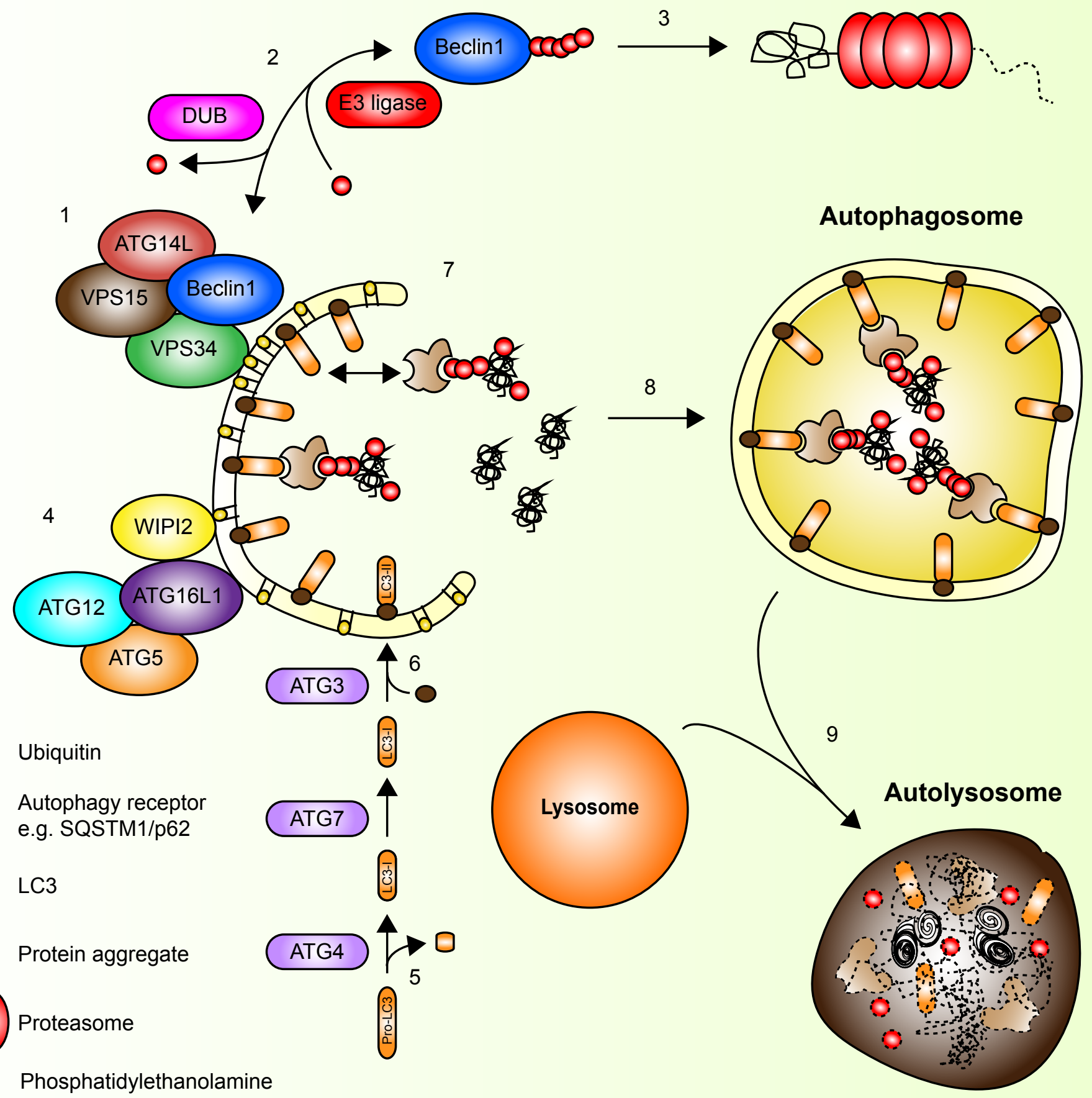

- Phosphatidylethanolamine

$\sigma \quad$ Phosphoinosityl-3-P 